Original Research Paper

\title{
Probing the Substrate Specificity and Intersubunit Interactions of Brevundimonas Diminuta Glutaryl Acylase with Site-Directed Mutagenesis
}

\author{
${ }^{1}$ Michael Eldarov, ${ }^{1}$ Anna Lyashenko, ${ }^{2}$ Tatyana Sherbakova, ${ }^{2}$ Dmitry Suplatov, ${ }^{2}$ KirillKopylov and ${ }^{2}$ Vytas Svedas \\ ${ }^{1}$ Centre "Bioengineering” Russian Academy of Sciences, Moscow, Russia \\ ${ }^{2}$ Belozersky Institute of Physicochemical Biology, Lomonosov Moscow State University, Moscow, Russia
}

Article history

Received: 2014-09-25

Revised: 2014-10-01

Accepted: 2014-11-07

Corresponding Auhtor: Michael Eldarov,

Centre "Bioengineering"

Russian Academy of

Sciences,

Moscow, Russia

Email: meldarov@mail.ru

\begin{abstract}
Glutaryl-7-ACA acylases (GLA) are industrial enzymes widely used for the production of 7-aminocepahlosporanic acid (7-ACA)-the starting compound for manufacturing of semisynthetic cephalosporin antibiotics. Generation of mutant GLA`s with increased activity and stability, capability for single-step conversion of cephalosporin $\mathrm{C}(\mathrm{CPC})$ directly to 7-ACA is a promising route to improve the current biocatalytic technologies. In this study GLA from Brevundimonas diminuta (BrdGLA) has been rationally re-designed to produce enzyme variants with improved properties. First, sequence analysis was performed to select residues responsible for substrate specificity as hotspots to introduce the capability to bind CPC in the active site of BrdGLA. Molecular modeling was used to evaluate the influence of selected residues on the formation of productive enzyme-substrate complex and the catalytic conversion. Genes, encoding mutant enzymes, were constructed, expressed and recombinant enzymes were purified on the chitin affinity resin. The mutant proteins showed induced catalytic activity against CPC. Second, BrdGLA mutants with increased activity and stability in alkaline conditions were obtained by mutating one of the surface lysine residues and replacement of the glutamine residue located in the active center by asparagine. Finally, structural analysis was used to select amino acid residues involved in formation of the quaternary structure of BrdGLA. Replacement of these "interface" positions to alanines led to a significant enzyme destabilization and reduction of its activity, confirming the role of the identified residues in the intersubunit interactions. The glutaraldehyde cross-linking has shown that the wild-type enzyme and its "interface" mutants possess complex oligomeric structure in solution with predominance of tetrameric forms.
\end{abstract}

Keywords: Cephalosporin C, Biocatalysis, Heterologous Expression, Molecular Modeling, Site-Directed Mutagenesis

\section{Introduction}

Acylases of glutaryl-7-aminocephalosporanic acid (Gl7ACA-acylase, EC 3.5.1.93, hereafter-glutaryl-acylases, GLA) are industrial enzymes extensively used for the production of 7-aminocephalosporanic acid (7-ACA)-the starting compound for manufacturing of semisynthetic cephalosporin antibiotics. Conversion of Cephalosporin $\mathrm{C}$ (CPC) to 7-ACA may be carried out either by chemical hydrolysis, requiring the use of highly toxic compounds and special reaction conditions at very low temperatures (Fechtig et al., 1968), or by enzymatic conversion (Sonawane, 2006). Currently known reactions of enzymatic CPC transformation can be divided into two groups: (i) Single-step conversion, using, for example, cephalosporin
C acylase from Pseudomonas (Conti et al., 2014) and (ii) two-step conversion, that implies the conversion of CPC to glutaryl-7-ACA using a D-amino acid oxidase (DAO; EC 1.4.3.3) from Trigonopsis variabilis (Szwajcer-Dey et al., 1990) or Rodotorula gracilis (Pollegioni et al., 1997) followed by GLA-catalyzed hydrolysis of the glutaryl-7ACA to form 7-ACA and glutarate (Conlon et al., 1995) or by enzymes of other classes belonging to the gammaglutamyltransferase (Yamada et al., 2008).

GLAs are of the most significant practical interest for biocatalytic synthesis of 7-ACA. GLAs are related to penicillin acylases. The latter are widely used in the industrial production of semisynthetic beta-lactam antibiotics (Srirangan et al., 2013). Similar to PAs, GLAs consist of two different subunits- $\alpha$ and $\beta$, but the 
three-dimensional structures of enzymes are different in the two families. PAs are heterodimeric whereas GLAs are heterotetramers composed of two heterodimers, which are formed from a single-stranded polypeptide precursor resulting from the-pecific processing. GLAs can effectively hydrolyze glutaryl-7-ACA, however cannot convert CPC. The exceptionally high biotechnological value of GLAs dictate the interest in the development of effective and efficient systems for production of these enzymes. We have previously developed efficient expression system for production of Brevundimonas diminuta GLA as a fusion with Nterminal chitin binding domain (BrdGLA/ChBD), enabling single-step purification of hybrid enzyme on the chitin-column (Khatuntseva et al., 2008). We have also shown that this modification does not affect protein activity and processing of GLA precursor and is even beneficial for protein thermal stability and catalytic properties. The developed expression/purification system provides an attractive opportunity for creation of novel GLA analogs to overcome several drawbacks of existing biocatalytic technologies. These drawbacks include, but are not limited to relatively low specific enzyme activity, its instability under the influence of mild denaturing agents and "crosslinking" agents, acidic and alkaline pH etc. (Monti et al., 2000).

The 3D structures of GLA precursor and mature processed form have been established (Kim et al., 2000), as well as the complex of this enzyme with the substrate and reaction products (Kim and Hol, 2001). These studies revealed important details of the structure of GLA active site, the mechanism of enzymatic reaction as well as autocatalytic enzyme processing and have opened the way to obtain various GLA analogs using methods of protein engineering. The main achievements in GLA protein engineering so far have been related to application of directed evolution to obtain variants with altered substrate specificity and site-directed mutagenesis of surface residues. Such variants are promising biocatalysts for production of 7aminodesacetoxycephalosporanic acid, an important intermediate in the synthesis of semisynthetic oral cephalosporins (Otten et al., 2002), and for development of more efficient methods of enzyme immobilization on various polymer carriers (Zhang et al., 2005).

Studies devoted to investigation of GLA quaternary structure are rather limited. GLA quaternary structure was reported to be rather labile (Battistel et al., 1998) and easily distorted by application of mild denaturants and extreme $\mathrm{pH}$ values. Generation of GLA analogs with more tight intersubunit interaction is a promising route towards enzyme stabilization. Other important unsolved issues are the identification of amino acid residues determining GLA substrate specificity, elucidation of enzyme kinetics and study of intersubunit interaction.
In this study GLA from Brevundimonas diminuta (BrdGLA) has been rationally re-designed to produce enzyme variants with improved properties. By using sequence and structure analysis we have obtained more active and stable GLA analogs as well as GLA variants capable for single-step conversion of CPC to 7-ACA. Furthermore, electrostatic interactions in the interface between the heterodimers were studied to evaluate the role of different amino acid residues in formation of the quaternary structure.

\section{Materials and Methods}

\section{Bacterial Strains and Plasmids}

Escherichia coli XL1-Blue [recA1 endA1 gyrA96 thi-1 hsdR17 supE44 relA1 lac [F'proAB lacIqZ $\Delta$ M15 Tn10 (Tetr)] (Stratagene, USA) was the host strain for the construction of BrdGLA/ChBD mutants. Mutant enzymes were expressed in E. coli BL21 (DE3) [FompThsdSB (rB- mB-) dcm gal dcm (DE3). The vector for expression of constructed BrdGLA/ChBD variants was pSVH0108, containing the gene for BrdGLA/ChBD fusion protein under the control of $\mathrm{T} 7$ promoter and terminator (Khatuntseva et al., 2010).

\section{DNA Manipulations}

Isolation of plasmid DNA, DNA hydrolysis with restriction endonucleases, isolation of DNA fragments from agarose gels, ligation of DNA fragments, PCR amplification was performed using enzymes and kits produced by "Sib Enzyme" (Russia), "Eurogen" (Russia) or "Fermentas MBI" (Lithuania)in accordance with the manufacturer's recommendations. DNA sequencing was performed using ABI BigDye3.1 sequencing kit (Applied Biosystems) according to the manufacturer's protocol on ABI3730 DNA sequencer. Site-directed mutagenesis was carried out using Quick-change sitedirected mutagenesis kit (Stratagene, USA). After final digestion with DpnI the reaction mixture was purified using CleanUp Mini kit (Eurogen, USA), 5 $\mu$ ls out of $15 \mu \mathrm{L}$ of purified sample were used to transform competent $E$. coli cells.

\section{Expression and Purification of BrdGLA/ChBD Variants}

E. coli BL21 (DE3) transformants, expressing various BrdGLA/ChBD variants were cultured in 100 $\mathrm{mL}$ of Overnight Express ${ }^{\mathrm{TM}}$ TB medium (Novagen, USA) with $50 \mu \mathrm{g} / \mathrm{ml}$ kanamycin in "auto induction" conditions for 36 hours at $28^{\circ} \mathrm{C}$. Cells were collected and suspended in $10 \mathrm{~mL}$ of Buffer A $(50 \mathrm{mM}$ sodium phosphate, $\mathrm{pH} 7,5,1 \mathrm{mM}$ EDTA, 0.3\% CTAB, 2\% glycerol) and disrupted by sonication. Crude cell homogenates were centrifuged at $8000 \mathrm{~g}, 4^{\circ} \mathrm{C}$ for 20 min. Resultant supernatants were purified by 30 to $65 \%$ 
saturation of ammonium sulfate at $4{ }^{\circ} \mathrm{C}$ to get protein samples containing recombinant enzymes. Precipitates were dissolved in buffer B $(50 \mathrm{mM}$ sodium phosphate, pH 7.5, 5 mM 2-mercaptoethanol, 2 mM EDTA, 10\% glycerol, $2 \mathrm{M} \mathrm{NaCl})$ and applied to a column $(1 \times 3 \mathrm{~cm})$ with chitin beads (New England Biolabs, USA) equilibrated with buffer B. The column was washed with the same buffer until the extinction of absorption at $280 \mathrm{~nm}$. Proteins specifically bound to the chitin column were eluted with Buffer $\mathrm{C}(50 \mathrm{mM}$ sodium phosphate, $\mathrm{pH} 7.5,1 \mathrm{mM}$ EDTA). The final enzyme preparations were concentrated by ultrafiltration on Amicon Ultra-15 centrifugal filter (Millipore) and assayed by SDS-PAGE. Protein concentration was determined by the method of (Bradford, 1976).

\section{Denaturing Gel Electrophoresis}

Electrophoresis under denaturing conditions was carried out according to the method of (Laemmli, 1970) using a $12 \%$ separating and $4 \%$ stacking polyacrylamide gels. For separation of protein samples cross linked with glutaraldehyde $7.5-20 \%$ denaturing gradient gel electrophoresis was used. The gel was poured so that the gel porosity increases from top to bottom.

\section{Enzyme Assays}

To determine the activity of BrdGLA/ChBD variants colorimetric method based on the reaction of chromophore formation upon interaction of 7-ACA amino group with p-dimethylbenzaldehyde was used as previously described (Khatuntseva et al., 2008). One unit of activity was determined as the amount of enzyme (in milligrams) required to convert $1 \mu$ mole of substrate during one minute of incubation. Specific activity was determined as the amount of enzyme ( $\mu$ moles of substrate per min at $37^{\circ} \mathrm{C}$ ) per $\mathrm{mg}$ of total protein in a sample. The total protein content was determined using method (Bradford, 1976).

\section{Determination of BrdGLA Stability}

To determine the stability of the wild-type enzyme and mutants thereof, samples with enzyme concentration of $43 \mu \mathrm{g} / \mathrm{ml}$ were incubated at $35^{\circ} \mathrm{C}$ in $0.1 \mathrm{M} \mathrm{K}$ phosphate buffer, $\mathrm{pH} 7.0-8.0$ or $0.05 \mathrm{M} \mathrm{K}$-phosphate buffer, $\mathrm{pH}$ 10.0. After distinct time intervals (from 1 hour to 3 days) enzyme aliquots were removed and activity was measured.

\section{Crosslinking of Enzyme Subunits with Glutaraldehyde}

Enzyme samples diluted in $50 \mathrm{mM}$ borate buffer $\mathrm{pH}$ 9.5 to a final concentration of $300 \mu \mathrm{g} / \mathrm{mL}$ were mixed with $1 / 5$ part of $1 \%$ aqueous solution of glutaraldehyde and the mixture was incubated for $40 \mathrm{~min}$ at $35^{\circ} \mathrm{C}$. The reaction was stopped by adding Tris-glycine buffer $(25$ mMTris, 200mm glycine, pH 8.3). Samples were kept at room temperature for $5 \mathrm{~min}$. Obtained samples were analyzed by electrophoresis under denaturing conditions.

\section{Molecular Modelling of the Full-Size Structure of BrdGLA/ChBD Fusion Protein}

Modelling of the full-size structure of $B$. diminuta GLA as a fusion protein with N-terminal chitin binding domain (BrdGLA/ChBD) was performed with Modeller v.9.12 (Šali and Blundell, 1993). High-resolution crystallographic structure of $B$. diminuta GLA in complex with glutaryl-7-aminocephalosporanic acid (PDB code 1JVZ) and the structure of ChBD domain from B.circulans chitinase A1 (PDB code 1ED7) were used as structural templates. The quality of the first 20 models was evaluated using the DOPE energy estimate, which is based on statistical comparison of obtained models with existing high-resolution crystallographic structures. The best candidate structure was selected and its geometry optimized by molecular modeling according to the protocols described earlier (Suplatov et al., 2014). The resulting structure contained BrdGLA with Nterminal chitin binding domain in complex with glutaryl7-aminocephalosporanic acid. The distances and angles of the crucial catalytic interactions in the active site satisfied the knowledge-based criteria of amidase catalytic activity (Radisky and Koshland, 2002). This molecular model of the enzyme-substrate complex was further used for computational research.

\section{Sequence and Structural Analysis of Substrate Specificity in BrdGLA}

To select positions responsible for substrate specificity of BrdGLA the following procedure was used. First, all residues within $5 \AA$ from the acyl moiety of the substrate were selected. Among these, the following residues were not further considered: Ser $\beta 1$ the catalytic nucleophile; Val $\beta 70$ and Asn $\beta 244$-oxyanion hole residues; Pro $\beta 22$-because its mutation can compromise structural organization; His $\beta 23$ and Thr $\beta 221$-because their side-chains are not involved in binding of the acyl moiety of the substrate. The remaining 9 positions were considered as hotspots for mutagenesis to design substrate specificity of BrdGLA to CPC. Sequence analysis was used to predict the amino acid substitutions at each selected site. Pfam alignment of the Ntn-hydrolase super family of enzymes (PFAM code 01804) was filtered for nonredundancy at $90 \%$ pair wise sequence identity. The resulting multiple alignment contained 845 protein sequences. Distribution of amino acid residues in the selected hotspots was evaluated. The following strategies were used to choose the particular amino acid substitutions at each selected position. First, the consensus approach was implemented, which implies that the most frequently observed amino acid types are favored 
by natural evolution and can be used to produce enzymes with improved properties (Lehmann et al., 2002). Therefore, the most popular amino acid types at each position were selected as candidate substitutions. Secondly, if the charged residues were present in other homologs in a corresponding position of the multiple alignments they were included into the list of mutations to accommodate the $\mathrm{D}$ - $\alpha$-amino adipyl moiety of $\mathrm{CPC}$ which contains both positively and negatively charged groups. Finally, mutation of large hydrophobic residues to smaller hydrophobic residues was considered to accommodate the larger acyl part of CPC. The following hotspots and mutations were selected (frequency of amino acid types in corresponding positions within the Ntn-hydrolase super family are shown in parenthesis): $\mathrm{Y} \alpha 149 \rightarrow \mathrm{L}(37 \%), \mathrm{F}(12 \%), \mathrm{R}(4 \%), \mathrm{D}(1 \%), \mathrm{K}(<1 \%)$, $\mathrm{E}(<1 \%) ; \mathrm{L} \beta 24 \rightarrow \mathrm{R}(10 \%), \mathrm{E}(<1 \%) ; \mathrm{F} \beta 31 \rightarrow \mathrm{S}(28 \%)$, $\mathrm{G}(13 \%), \quad \mathrm{V}(3 \%), \quad \mathrm{L}(3 \%), \quad \mathrm{R}(2 \%), \quad \mathrm{D}(2 \%), \quad \mathrm{E}(1 \%)$, $\mathrm{K}(<1 \%) ; \mathrm{Y} \beta 33 \rightarrow \mathrm{W}(49 \%), \mathrm{R}(6 \%) ; \mathrm{Q} \beta 50 \rightarrow \mathrm{L}(31 \%)$, $\mathrm{F}(24 \%), \mathrm{E}(5 \%), \mathrm{R}(<1 \%), \mathrm{H}(<1 \%) ; \mathrm{R} \beta 57 \rightarrow \mathrm{V}(20 \%)$, $\mathrm{L}(16 \%), \mathrm{I}(14 \%), \mathrm{H}(5 \%), \mathrm{E}(2 \%), \mathrm{R}(<1 \%) ; \mathrm{F} \beta 58 \rightarrow$ $\mathrm{I}(36 \%), \mathrm{L}(18 \%), \mathrm{V}(9 \%), \mathrm{H}(1 \%), \mathrm{D}(<1 \%), \mathrm{E}(<1 \%)$; $\mathrm{N} \beta 68 \rightarrow \mathrm{H}(18 \%), \mathrm{L}(15 \%), \mathrm{R}(<1 \%), \mathrm{E}(<1 \%) ; \mathrm{F} \beta 177 \rightarrow$ $\mathrm{Q}(32 \%), \mathrm{L}(12 \%), \mathrm{V}(11 \%), \mathrm{E}(4 \%), \mathrm{H}(<1 \%), \mathrm{D}(<1 \%)$. The role of the selected residues was further evaluated by molecular modeling.

Molecular Modeling of Substrate Specificity in BrdGLA and Screening of in Silico library of Mutants

Molecular models of BrdGLA variants with substitutions suggested by sequence and structure analysis were generated and screened by molecular docking using $\mathrm{CPC}$ as the substrate. All single mutants, as well as all double and triple combinations of the selected mutations were considered. Protocols for molecular docking with the Auto dock software (Huey et al., 2007) and preparation of in silico libraries were discussed previously (Suplatov et al., 2012). Structural filtration of the resulting enzymesubstrate complexes was used as a post-docking tool to select mutants that were able to stabilize the productive binding state in the active site. Distances of crucial catalytic interactions that describe trajectory of the nucleophilic attack were restricted as follows. Distance between the carbonyl carbon atom of the substrate and the $\gamma$-oxygen catalytic Ser $\beta 1$, as well as distances between the substrate's carbonyl oxygen and main-chain of Val $\beta 70$ or side-chain of Asn $\beta 244$-were limited to $3.5 \AA$. In addition, the absolute values of angle and dihedral angle of a nucleophilic attack trajectory of the Ser $\beta 1 \gamma$-oxygen on 80 to $100^{\circ}$ (Radisky and Koshland, 2002). Enzyme-substrate complexes that were out of range were dismissed. The final results were ranked by the frequency of the productive binding geometry (how many times the near-to-attack conformation was observed after 100 independent docking runs). The first top-ranked mutant was further evaluated experimentally. Scientific illustrations of biological macromolecules were prepared with PyMol software.

\section{In Silico study of the BrdGLA Quaternary Structure}

Crystal structure of a heterotetrameric structure of GLA from Pseudomonas sp. 130 (PDB code 4E56) was used as a template to model the quaternary of BrdGLA/ChBD.

\section{Results and Discussion}

\section{BrdGLA Variants with Altered Substrate Specificity}

Structural analysis of the active site of BrdGLA in a complex with glutaryl-7-aminocephalosporanic acid suggested three sub-sites for binding of different functional moieties of the substrate: (1) The acyl moiety, which is the substituent at the $\mathrm{C} 7$ carbon atom of the $\beta$ lactam ring; (2) the $\beta$-lactam ring and (3) substituents at the six-membered cycle of the $\beta$-lactam ring. The most intense intermolecular interactions were observed in the binding site of the acyl moiety of the substrate: Tyro149, $\operatorname{Tyr} \beta 33$, Gln $\beta 50, \operatorname{Arg} \beta 57$ stabilize the negative charge on the carboxyl group, while Leuß24, Phe $\beta 58$, Val $\beta 70$ and Phe $\beta 177$ form a hydrophobic environment for the atoms C9, C5 and C17 of the glutaryl moiety. These interactions determine specificity of the enzyme towards the native substrate (Fig. 1).

To extend the substrate specificity of BrdGLA we have re-designed the active site to accommodate the CPC. Compared to glutarylmoiety of G17ADCA, the D$\alpha$-amino- $\delta$-adipyl moiety of CPC contains both positively and negatively charged groups and has a larger hydrophobic side-chain. Therefore, the active site should contain a positively charged amino acid residue for binding of the carboxyl group and also a negatively charged as partate or glutamate to stabilize the positively charged amino group. The corresponding in silico library of BrdGLA variants was generated and screened. Since the BrdGLA binding site lacs as partate or glutamate residues in the active site, the respective counter ion was introduced as well as the overall volume of the bind cleft was increased by substitutions to smaller hydrophobic residues. The triple mutant Y $\alpha 149 \mathrm{E} / \mathrm{F} \beta 31 \mathrm{~L} / \mathrm{F} \beta 177 \mathrm{~L}$ was found to be the best in accommodating the proper binding and the near-to-attack conformation of the substrate in the active site. Structural analysis of the resulting enzyme-substrate complex showed that CPC binding at the active site of the mutant enzyme is similar to binding of G17ACA in the active site of the wild-type protein (Fig. 2). The acyl moiety binds inside a deep cone-shaped cavity which has charged and polar amino acid residues located at the bottom. 


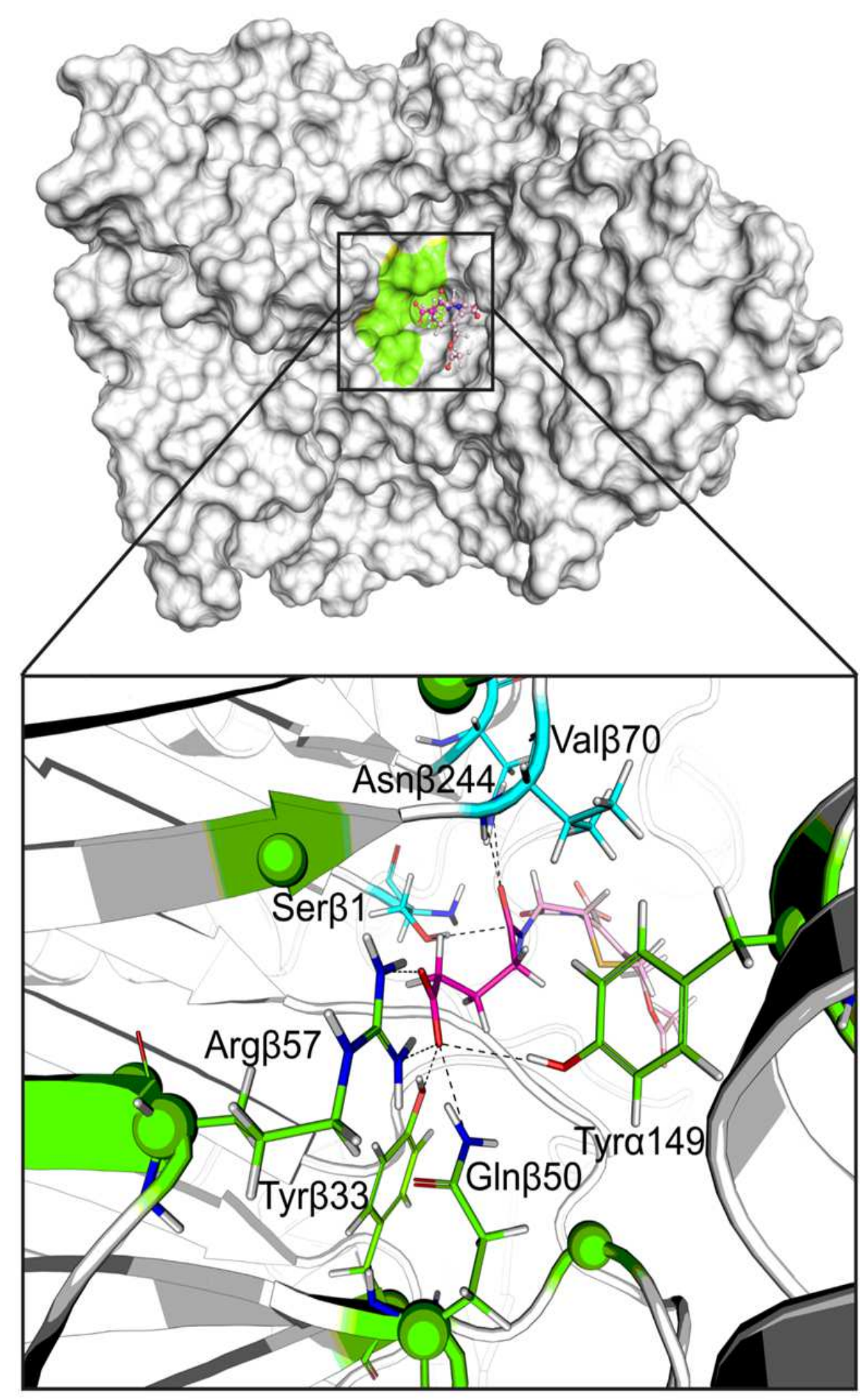

Fig. 1. BrdGLA in a complex with glutaryl-7-aminocephalosporanic acid. Glutaryl moiety of the substrate is colored in magenta. Dashed lines indicate electrostatic interactions between the active site residues and the substrate. $\mathrm{C} \alpha$-atoms of residues selected as hotspots for mutagenesis are shown as green spheres 


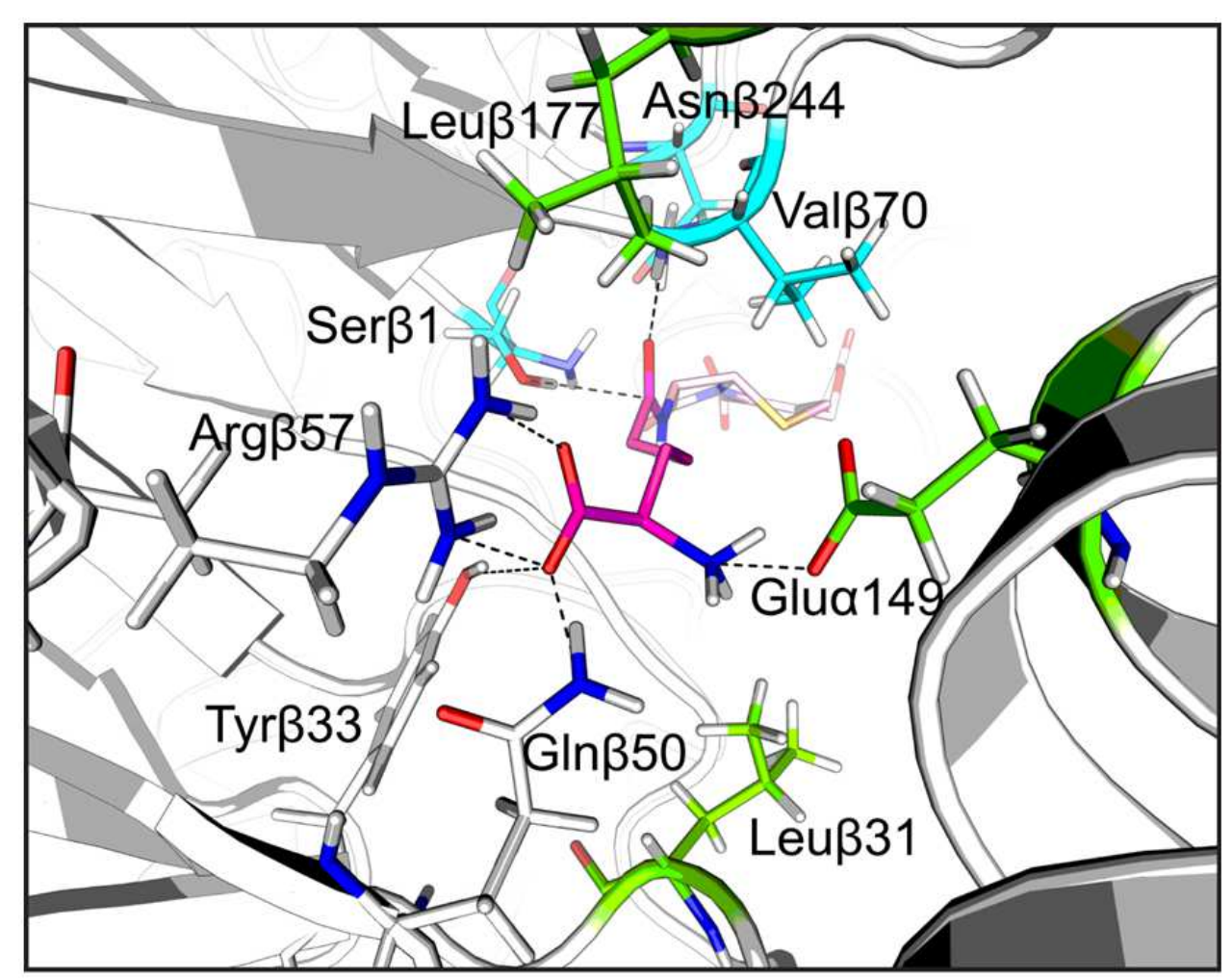

Fig. 2. BrdGLA variant Y $\alpha 149 \mathrm{E} / \mathrm{F} \beta 31 \mathrm{~L} / \mathrm{F} \beta 177 \mathrm{~L}$ in a complex with cephalosporin C. D- $\alpha$-amino adipyl moiety of CPC is colored in magenta. Residues introduced by point mutagenesis are colored in green. Dashed lines indicate electrostatic interactions between the active site residues and the substrate

Tyr $\beta 33, G \ln \beta 50$ and $\operatorname{Arg} \beta 57$ residues interact with carboxylate of the $\mathrm{D}$ - $\alpha$-amino- $\delta$-adipyl moiety of the substrate, which adopts orientation similar to the carboxylate of the glutaryl moiety of the G17ADCA. At the same time, Y $\alpha 149 \mathrm{E}$ accommodates the positively charged amino group of the CPC's acyl side-chain. The binding of the $\beta$-lactam ring, as well as its substituents occurs similar to G17ACA.

Based on the results of sequence and structure analysis the following single, double and triple BrdGLA variants were produced: Y $\alpha 149 \mathrm{E}, \mathrm{F} \beta 177 \mathrm{~L}$, $\mathrm{Y} \alpha 149 \mathrm{E} / \mathrm{F} \beta 31 \mathrm{~L}$ and $\mathrm{Y} \alpha 149 \mathrm{E} / \mathrm{F} \beta 31 \mathrm{~L} / \mathrm{F} \beta 177 \mathrm{~L}$. Kinetic parameters of affinity-purified BrdGLA/ChBD variants were determined using G17ACA and CPC as substrates (Table 1). The highest activity towards G17ACA was detected for the wild-type enzyme. The triple mutant showed lowest activity with the native substrate. The activity of all enzyme variants towards CPC was improved compared to the wild-type enzyme; however appeared to be low in general. The activity of the triple mutant with CPC constituted not more than $0.3 \%$ of the activity of wild-type enzyme with the natural substrate G17ACA. The reason for such low performance can be explained by the inability of the mutant enzymes to hydrolyze CPC due to the problems with binding of the cephalosporin nucleus. To overcome this limitation, additional efforts in re-designing the active site residues may be required. Nonetheless, obtained results show that the employed strategy can be used to extend substrate specificity of BrdGLA towards CPC.

When this article was in preparation we became aware of the very successful attempt to engineer GLA from Pseudomonas N176 towards mono-step process for CPC conversion (Conti et al., 2014). By combining structural knowledge, semi-rational design and evolution analysis authors were able to isolate enzyme variants with more than 11,000-fold increase in specificity constant and with the highest kinetic efficiency so far obtained for a cephalosporin acylase.

\section{Site-directed Mutagenesis to Enhance BrdGLAStability and Activity}

Previously it was shown that introduction of the double K $\beta 198 \mathrm{~A} / \mathrm{Q} \beta 50 \mathrm{~N}$ mutation in Pseudomonas 130 GLA increases enzyme stability and activity (Zhang et al., 2005). We have introduced orthologous mutation in our BrdGLA/ChBD fusion protein and have studied properties of the mutant enzyme. Replacement of glutamine with asparagine at position 50 is expected to enlarge the substrate binding site and lead to stronger GL7ACA binding in the substrate pocket. 
Table 1. Specific activity of BrdGLA mutants in enzymatic hydrolysis of GL7ACA and CPC

\begin{tabular}{|c|c|c|c|}
\hline \multirow[b]{2}{*}{ Enzyme } & \multicolumn{2}{|l|}{ G17ACA } & \multirow{2}{*}{$\begin{array}{l}\text { CPC } \\
\begin{array}{l}\text { Specific activity } \\
\left(\mu \mathrm{mole}^{*} \mathrm{~min}^{-1} * \mathrm{mg}^{-1}\right)\end{array}\end{array}$} \\
\hline & $\begin{array}{l}\text { Specific activity } \\
\left(\mu \text { mole }^{*} \min ^{-1} * \mathrm{mg}^{-1}\right)\end{array}$ & $\begin{array}{l}\text { Compared to activity } \\
\text { of wild type BrdGLA (\%) }\end{array}$ & \\
\hline BrdGLA wild type & 8.750 & 100 & 0.0023 \\
\hline BrdGLA F $\beta 177 \mathrm{~L}$ & 0.770 & 8.8 & 0.0091 \\
\hline BrdGLA Y $\alpha 149 \mathrm{E}$ & 0.380 & 4.3 & 0.0069 \\
\hline BrdGLA Y $\alpha 149 \mathrm{E} / \mathrm{F} \beta 31 \mathrm{~L}$ & 0.520 & 5.9 & 0.0110 \\
\hline 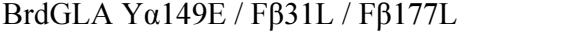 & 0.045 & 0.5 & 0.0230 \\
\hline BrdGLAY $\alpha 149 \mathrm{E} / \mathrm{F} \beta 31 \mathrm{~L} / \mathrm{F} \beta 58 \mathrm{~L} / \mathrm{F} \beta 177 \mathrm{~L}$ & n.d. & n.d. & n.d. \\
\hline
\end{tabular}

Substitution of the surface lysine residue at position $\beta 198$ with alanine should stabilize the tetramer structure. The activity of the double mutant measured in optimal conditions $\left(35^{\circ} \mathrm{C}, \mathrm{pH} 7.0\right)$ did not differ from wild type enzyme and constituted $7 \pm 0.5 \mu \mathrm{moL}^{*} \mathrm{~min}^{-1} * \mathrm{mg}^{-1}$. The stability of the double-mutant at $\mathrm{pH}$ optimal for the wild type protein also did not increase: after 70 hours of incubation at $35^{\circ} \mathrm{C}$ and $\mathrm{pH} 7.0$ the residual activity of the mutant protein was just $15 \%$ lower than the activity of the wild-type protein. Taking into account the reported influence of the Q $\beta 50 \mathrm{~N}$ mutation on $\mathrm{pH}$-optimum of mutant enzyme and its stability at alkaline conditions (Zhang et al., 2005) we have studied the rate of thermal inactivation of the double mutant at $\mathrm{pH}$ 10.0. Results in comparison with previous experiment are shown on Fig. 3. It is obvious that both the mutant and the wild type enzyme are more stable at neutral pH (Fig. 3, lines 1 and 2) than at alkaline conditions (Fig. 3, lines 3 and 4). However, at pH 10.0 the mutant (Fig. 3 , line 3 ) is $30 \%$ more stable than the wild type enzyme (Fig. 3, line 4) under the same conditions. Improved enzyme stability at alkaline $\mathrm{pH}$ accompanied with the high level of catalytic activity is favorable for industrial biocatalysis, since it overcomes partial enzymes inactivation that may occur at micro alkaline environment in the course of enzymatic 7-ACA production.

\section{Investigation of BrdGLA oligomeric State and the Analysis of the Dimer-Dimer Interface}

The data concerning the quaternary structure of GLAs is contradictory. According to the results of biochemical studies enzymes are tetramers (Battistel et al., 1998). At the same time, in the majority of publicly available 3D structures GLAs are represented mainly by $\alpha / \beta$ dimers, the fact that may be related to peculiarities of enzyme crystallization. In the recently obtained structures of unprocessed GLA precursor the protein is presented as a dimer of two fused $\alpha / \beta$ chains (PDB code 4E56). We have used this structure to study amino acid residues forming the interface between the two $\alpha / \beta$ dimers. Two clusters of intersubunit contacts were further studied experimentally.

\section{Alpha-Subunit Mutants}

Structural analysis revealed that amino acid residues Gln $\alpha 91, \operatorname{Trp} \alpha 92, \operatorname{Asp} \alpha 120, \operatorname{Asp} \alpha 121$ and
Ile $\alpha 122$ within the $\alpha$-subunit participate in the formation of salt bridges, hydrogen and hydrophobic bonds between the two $\alpha / \beta$ dimers (Fig. 4).

To analyze the role of these residues the corresponding alanine mutants were produced - Q $\alpha 91 \mathrm{~A} / \mathrm{W} \alpha 92 \mathrm{~A}$, $\mathrm{D} \alpha 121 \mathrm{~A} / \mathrm{I} \alpha 122 \mathrm{~A}, \mathrm{D} \alpha 120 \mathrm{~A} / \mathrm{D} \alpha 121 \mathrm{~A} / \mathrm{I} \alpha 122 \mathrm{~A}$. The mutant proteins were expressed in E.coli cells using the standard protocol and recombinant enzymes were affinity-purified on chitin sorbent. Kinetic parameters of purified protein preparations were determined using G17ACA as substrate. The highest activity was observed for the double D $121 \mathrm{~A} / \mathrm{I} \alpha 122 \mathrm{~A}$ mutant $\left(11 \mu \mathrm{mole}^{*} \mathrm{~min}^{-1} * \mathrm{mg}^{-1}\right)$, while the activity of the triple $\mathrm{D} \alpha 120 \mathrm{~A} / \mathrm{D} \alpha 121 \mathrm{~A} / \mathrm{I} \alpha 122 \mathrm{~A}$ mutant was the lowest $\left(2 \mu\right.$ mole* $\left.\min ^{-1} * \mathrm{mg}^{-1}\right)$. The activity of the $\mathrm{Q} \alpha 91 \mathrm{~A} / \mathrm{W} \alpha 92 \mathrm{~A}$ mutant constituted 5.4 umole* $\mathrm{min}^{-1} * \mathrm{mg}^{-1}$. This implies the crucial role of the D 120 . We assume that the decrease of activity of the triple mutant is due to the destabilization of the BrdGLA tetrameric structure, which leads to dissociation of functionally active tetramer to inactive dimers. Additional experiments were conducted to get more insight into oligomeric state of BrdGLA. In particular, we studied the stability of the mutants under neutral conditions at $37^{\circ} \mathrm{C}$. Results are presented in Fig. 5. Introduction of the described $\alpha$-subunit mutations led to the decrease in stability of all mutants as compared to the wild-type enzyme. However, the nature of the stability change was different for different mutants and ambiguously correlated with the data on enzyme activity. For example the activity of the double Q $\alpha 91 \mathrm{~A} / \mathrm{W} \alpha 92 \mathrm{~A}$ mutant was insignificantly decreased and constituted $71 \%$ of the wild type enzyme. At the same time, this mutant showed the worst stability: $80 \%$ of activity was lost after $20 \mathrm{~h}$ of incubation (Fig. 5, line 4). Interestingly, the rate of inactivation of the double Do121A/I $\alpha 122 \mathrm{~A}$ mutant (Fig. 5, line 2), that has the highest activity, is slower than that of the triple mutant (Fig. 5, line 3): After 45 hours of incubation the double mutant retains $74 \%$ of its original activity, whereas the activity of the triple mutant drops to $52 \%$. Thus, the values of mutant stability correlate well with the values of their activity.

In general, the correlation between mutant stability and activity is rather good and corresponds well to the predicted role of the replaced residues in the formation of the intersubunit contacts. In all cases, the substitution of amino acid residues located in the proposed interface area to alanine results in enzyme destabilization. 


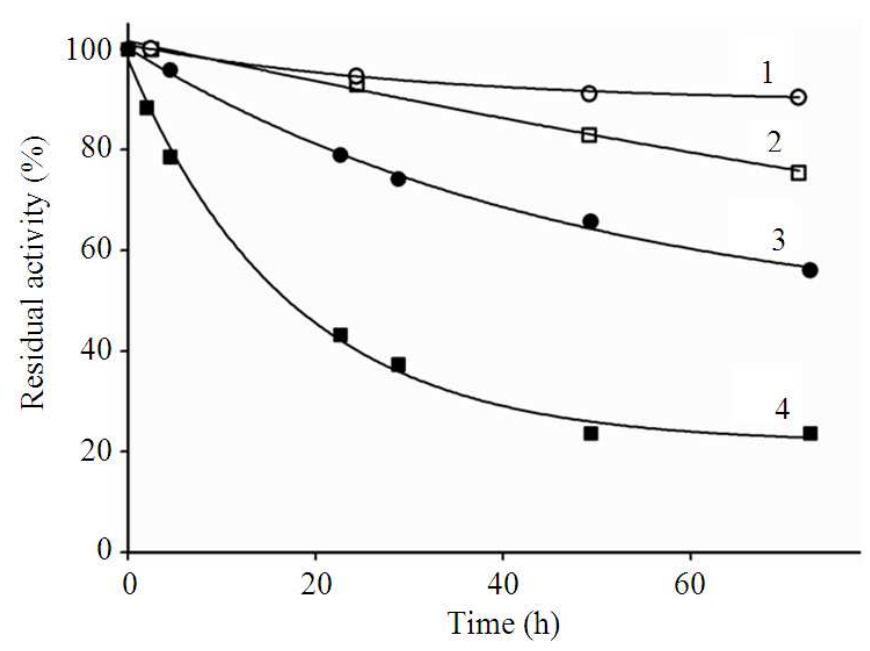

Fig. 3. Stability of the wild type of BrdGLA (lines 1 and 4) and the K $\beta 198 \mathrm{~A} / \mathrm{Q} \beta 50$ Nmutant of BrdGLA (lines 2 and 3 ) at neutral $(\mathrm{pH} \mathrm{7.0,} \mathrm{lines} 1$ and 2$)$ and alkaline $(\mathrm{pH} 10.0$, lines 3 and 4$)$ conditions at $35^{\circ} \mathrm{C}$

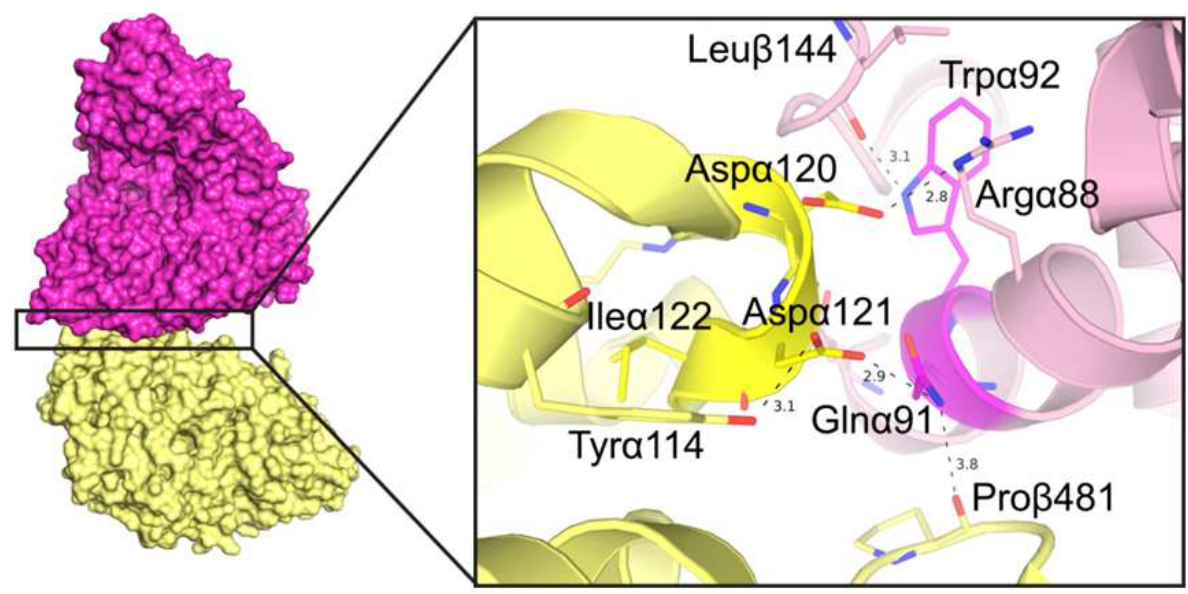

Fig. 4. Interface area between the two $\alpha / \beta$ dimers of the BrdGLAheterotetramer

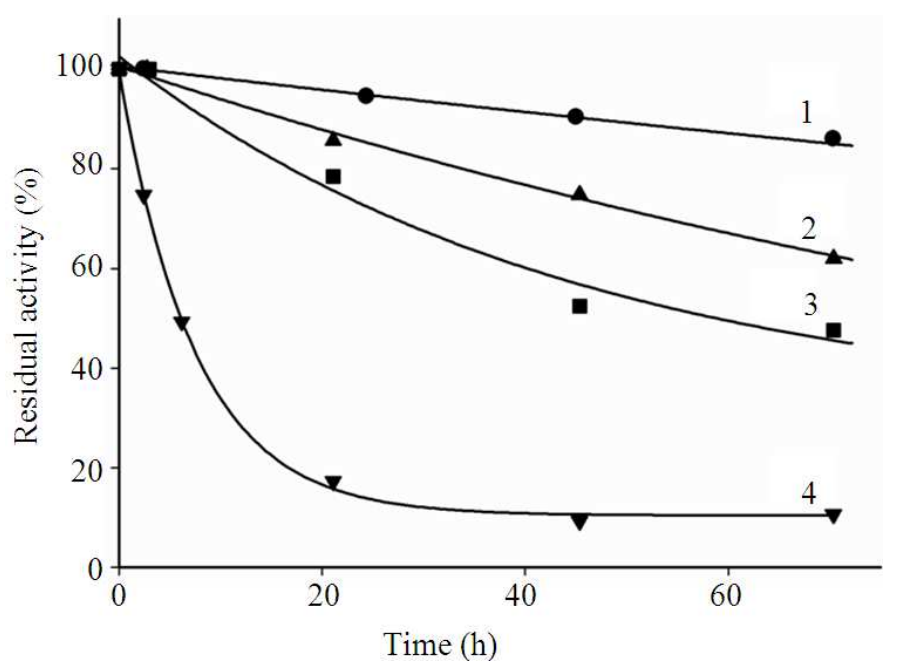

Fig. 5. Stability of the $\alpha$-subunit mutants of BrdGLA at neutral conditions $\left(0.1 \mathrm{M} \mathrm{KH}_{2} \mathrm{PO}_{4}, \mathrm{pH} 8.0,37^{\circ} \mathrm{C}\right)$ : 1-the wild type BrdGLA, 2-the $\mathrm{D} \alpha 120 \mathrm{~A} / \mathrm{D} \alpha 121 \mathrm{~A} / \mathrm{I} \alpha 122 \mathrm{~A}$ mutant, 3-the D $\alpha 121 \mathrm{~A} / \mathrm{I} \alpha 122 \mathrm{~A}$ mutant, 4- the $\mathrm{Q} \alpha 91 \mathrm{~A} / \mathrm{W} \alpha 92 \mathrm{~A}$ mutant 
Most mutants possess lower activity, indicating important role of the tetramer for BrdGLA functionality. Partial increase in the activity in the double $\mathrm{D} \alpha 121 \mathrm{~A} / \mathrm{I} \alpha 122 \mathrm{~A}$ mutant may be due to the greater availability of the active center. Introduction of the third $\mathrm{D} \alpha 120 \mathrm{~A}$ mutation leads to lowering of activity and stability values. This implies that this residue is critical for the maintenance of functional, stable state of the enzyme.

\section{Mutants of Beta-Subunit}

The third region of the dimer-dimer interface comprises four consecutive amino acid residues within the $\beta$-strand in the $\mathrm{C}$-terminus of the $\beta$ subunit. These residues are important for ionic interactions and hydrogen bonds with the part of the other dimer containing several adjacent positively and negatively charged amino acids. To elucidate the role of this site we have obtained a series of BrdGLA/ChBD deletion variants by successive elimination of $\mathrm{C}$-terminal amino acids comprising this region. Five constructs were obtained:

- 1-d749-753-carries a deletion of the five terminal amino acids: FNFLP

- 2-d748-753-carries a deletion of the six terminal amino acids: PFNFLP

- 3-d747-753-carries a deletion of seven terminal amino acids: TPFNFLP

- 4-d746-753-carries a deletion of eight terminal amino acids: RTPFNFLP

- 5-d744-753-carries a deletion of ten terminal amino acids: QERTPFNFLP

Recombinant BrdGLA/ChBD variants were expressed and purified using the standard protocol. Proteins with most significant deletions (more than 6 C-terminal amino acids) could not be isolated. The yield of two successfully purified variants d749-753 and d748-753 comprised $200 \mu \mathrm{g}$ from $30 \mathrm{ml}$ culture, which is five times less than that of $\alpha$-subunit mutants. The specific activity of d748-753 and d749753 mutants decreased to 2.6 and $3.0 \mu \mathrm{mol}^{*} \mathrm{~min}^{-}$ ${ }^{1} * \mathrm{mg}^{-1}$ respectively, constituting 35 and $40 \%$ of the activity of the wild-type enzyme (Table 2). Stability measurements have shown that the C-terminal mutants are generally less stable than the wild-type enzyme, but their stability is higher than that of the $\alpha$ subunit mutants. After incubation during 45 hours the activity of $\mathrm{d} 748-753$ and $\mathrm{d} 749-753$ mutants dropped to $78 \%$ and $64 \%$, respectively. Thus, the mutant with deeper deletion $\mathrm{d} 748-753$ has a lower activity compared to the d749-753 mutant and simultaneously the higher stability. Despite the lack of direct correlation between the activity and stability of the two mutants studied, there is no doubt that the introduced deletions resulted in significant protein destabilization and inactivation, consistent with the proposed role of the $C$-terminal region of the $\beta$ subunit in tetramer formation.

\section{Modification of BrdGLA/ChBD with Glutaraldehyde}

Intermolecular cross-linking of protein subunits with different cross-linking agents is a useful method to investigate the protein oligomeric state. We have used the glutaraldehyde cross linking to ascertain the subunit composition of the BrdGLA/ChBD. As evident from the electrophoregram presented on Fig. 6, a large number of additional bands is present in the sample obtained after cross-linking. Besides major bands corresponding to $\alpha$ - and $\beta$-subunits (20 and $50 \mathrm{kDa}$ ) a distinct band of $80 \mathrm{kDa}$, corresponding probably to protein dimer and $115 \mathrm{kDa}$ band, likely corresponding to heterotetramer, can be easily identified. Intense distinct higher molecular weight bands (240 kDa and above) are also present. We assume that the $240 \mathrm{kDa}$ band corresponds to protein octamer. Numerous minor bands likely arise due to arbitrary cross-links between the $\alpha-\beta$ dimer and $\alpha$-subunit, $\alpha-\beta$ dimer and $\beta$-subunit etc. In general, experiment with glutaraldehyde has shown the abundance of oligomeric forms generated as a result of cross-linking between protein subunits and separate oligomers. The presence of tetramers and higher molecular weight forms generated as results of intermolecular cross linking may indicate the predominance of tetramers in solution of the native enzyme.

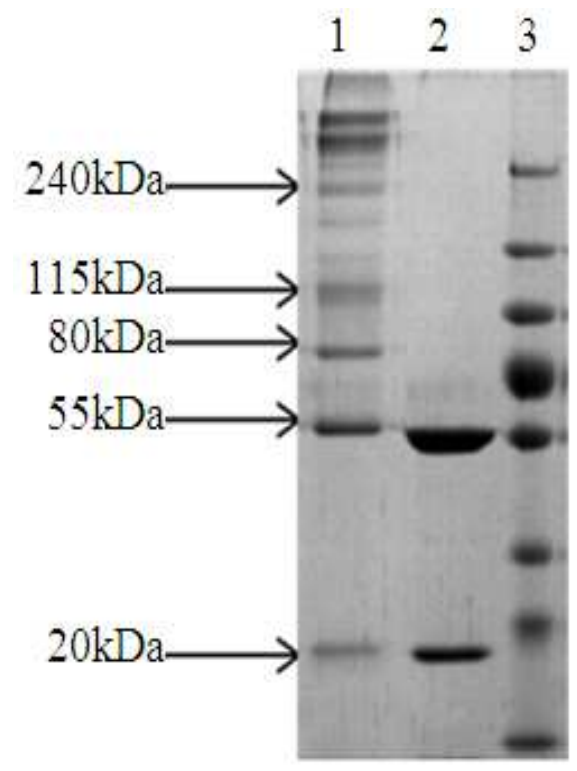

Fig. 6. Separation of crosslinkedBrdGLA/ChBDprotein in a gradient SDS-PAGE 
Table 2. Activity and stability of the wild type enzyme and double mutant BrdGLA K $\beta 198 \mathrm{~A} / \mathrm{Q} \beta 50 \mathrm{~N}$ at neutral and alkaline $\mathrm{pH}$

\begin{tabular}{|c|c|c|c|}
\hline \multirow[b]{2}{*}{ Mutant } & \multirow[b]{2}{*}{$\begin{array}{l}\text { Specific activity }\left(\mu \mathrm{mole}^{*} \mathrm{~min}^{-1} * \mathrm{mg}^{-1}\right) \\
\text { pH } 7.0\end{array}$} & \multicolumn{2}{|c|}{ Residualactivity $70 \mathrm{~h}, 35^{\circ} \mathrm{C}(\%)$} \\
\hline & & $\mathrm{pH} 7.000$ & pH 10.0 \\
\hline BrdGLA wild type & 7.6 & 90.000 & 23.0 \\
\hline BrdGLAK $\beta 198 \mathrm{~A} / \mathrm{Q} \beta 50 \mathrm{~N}$ & 7.2 & 75.000 & 56.0 \\
\hline
\end{tabular}

\section{Conclusion}

Amino acid residues critical for substrate specificity of B. diminuta glutaryl acylase were identified using sequence and structure analysis, molecular modeling and site-directed mutagenesis. The chosen strategy of bioengineering of BrdGLA aimed at changing its substrate specificity have enabled to induce new enzymatic activity and obtain enzyme variants with catalytic activity against cephalosporin C. Through modelling of BrdGLA intersubunit interactions amino acid residues forming contacts between two $\alpha-\beta$ dimers have been identified. Variants of BrdGLA with replacement of these amino acid residues to alanine are characterized by reduced stability and diminished activity, proving the role of these residues in formation of the contact area between two $\alpha-\beta$ dimers. Protein cross-linking experiments have shown that various oligomeric forms coexist in the solution with predominance of tetramers. Finally, the double $\mathrm{Q} \beta 50 \mathrm{~N} / \mathrm{K} \beta 198 \mathrm{~A}$ BrdGLA mutant was obtained that is promising for development of more efficient biocatalysts stable in alkaline medium.

\section{Acknowledgement}

Lomonosov Moscow State University supercomputer cluster 'Lomonosov' was used for bioinformatic calculations (Voevodin et al., 2012).

\section{Funding Information}

This study was supported by the Russian Foundation for Basic Research (Grant\# 12-08-01163). The research of M. Eldarov at Centre "Bioengineering" RAS was supported in part by the Ministry of Education and Science of Russian Federation is as follows: Project ID RFMEFI60414X0022).

\section{Author's Contributions}

Michael Eldarov: Conceived of the research, designed the study, drafted the paper.

Anna Lyashenko: Performed mutagenesis, protein expression and purification.

Tatyana Sherbakova: Performed activity measurements, protein cross-linking, helped with manuscript preparation.

Dmitry Suplatov and Kirill Kopylov:

Were responsible for computer modeling, in silico design of mutations, finalized the paper.

Vytas Svedas: Was general supervisor and revised the article.

\section{Ethics}

We confirm that this manuscript has not been published elsewhere and is not under consideration by another journal. Each author confirms the manuscript represents honest work. All authors have approved the manuscript. Each author agrees with the order in which his name appears on the title page.

\section{References}

Battistel, E., D. Bianchi, R. Bortolo and L. Bonoldi, 1998. Purification and stability of glutaryl-7-ACA acylase from Pseudomonas sp. Applied Biochem. Biotechnol., 69: 53-67. DOI: $10.1007 / \mathrm{BF} 02786021$

Bradford, M.M., 1976. A rapid and sensitive method for the quantitation of microgram quantities of protein utilizing the principle of protein-dye binding. Anal. Biochem., 72: 248-254. DOI: 10.1016/0003-2697(76)90527-3

Conlon, H.D., J. Baqai, K. Baker, Y.Q. Shen and B.L. Wong et al., 1995. Two-step immobilized enzyme conversion of cephalosporin $\mathrm{C}$ to 7aminocephalosporanic acid. Biotechnol. Bioeng., 46: 510-513. DOI: 10.1002/bit.260460603

Conti, G., L. Pollegioni, G. Molla and E. Rosini. 2014. Strategic manipulation of an industrial biocatalyst evolution of a cephalosporin C acylase. FEBS J., 281: 2443-2455. DOI: 10.1111/febs. 12798

Fechtig, B., H. Peter, H. Bickel and E. Vischer. 1968. Modification of antibiotics. 11. Preparation of 7aminocephalosporanic acid. Helv. Chim. Acta., 51: 1109-1120.

Huey, R., G.M. Morris, A.J. Olson and D.S. Goodsell, 2007. A semiempirical free energy force field with charge-based desolvation. J. Comput. Chem., 28: 1145-1152.

Khatuntseva, S.A., M.A. Ehl'darov, O.A. Zeinalov and K.G. Skrjabin, 2010. Recombinant DNA, which codes functionally active hybrid protein GL7ACA-acylase with chitin-binding domain (BrdGL7ACA-cbd) and recombinant plasmid pSVH0108, providing for its synthesis in cells Escherichia coli, recombinant strain Escherichia coli BL21 (DE3)/pSVH0108-PRODUCER BrdGL7ACA-cbd. Patent of the Russian Federation 2388826. 
Khatuntseva, S.A., M.A. Eldarov, V.A. Redo and K.G. Skryabin, 2008. Purification and immobilization of recombinant variants of Brevundimonas diminuta glutaryl-7-aminocephalosporanic acid acylase expressed in Escherichia coli cells. J. Biotechnol., 133: 123-126. DOI: 10.1016/j.jbiotec.2007.08.044

Kim, Y. and W.G. Hol, 2001. Structure of cephalosporin acylase in complex with glutaryl-7aminocephalosporanic acid and glutarate: Insight into the basis of its substrate specificity. Chem. Biol., 8: 1253-1264. DOI: 10.1016/S1074-5521(01)0009-8

Kim, Y., K. Yoon, Y. Khang, S. Turley and W.G. Hol, 2000. The 2.0 a crystal structure of cephalosporin acylase. Structure, 8: 1059-1068.

DOI: $10.1016 / \mathrm{S} 0969-2126(00) 00505-0$

Laemmli, U.K. 1970. Cleavage of structural proteins during the assembly of the head of bacteriophage T4. Nature, 227: 680-685. DOI: 10.1038/227680a0

Lehmann, M., C. Loch, A. Middendorf and D. Studer et al., 2002. The consensus concept for thermostability engineering of proteins: Further proof of concept. Protein Eng., 15: 403-411. DOI: $10.1093 /$ protein/15.5.403

Monti, D., G. Carrea, S. Riva, E. Baldaro and G. Frare, 2000. Characterization of an industrial biocatalyst: Immobilized glutaryl-7-ACA acylase. Biotechnol. Bioeng., 70: 239-244. DOI: 10.1002/10970290(20001020)70:2<239::AID-BIT13>3.0.CO;2-I

Otten, L.G., C.F. Sio, J. Vrielink, R.H. Cool and W.J. Quax. 2002. Altering the substrate specificity of cephalosporin acylase by directed evolution of the beta-subunit. J. Biol. Chem., 277: 42121-42127. DOI: $10.1074 /$ jbc.M208317200

Pollegioni, L., G. Molla, S. Campaner, E. Martegani and M.S. Pilone, 1997. Cloning, sequencing and expression in E. coli of a D-amino acid oxidase cDNA from Rhodotorulagracilis active on cephalosporin C. J. Biotechnol., 17: 115-123. DOI: 10.1016/S0168-1656(97)00142-9.

Radisky, E.S. and D.E. Koshland, 2002. A clogged gutter mechanism for protease inhibitors. Proc. Natl. Acad. Sci. U.S.A., 99: 10316-10321.

Šali, A. and T.L. Blundell, 1993. Comparative protein modelling by satisfaction of spatial restraints. J. Molecul. Biol., 234: 779-815.

DOI: $10.1006 /$ jmbi.1993.1626
Sonawane, V.C., 2006. Enzymatic modifications of cephalosporins by cephalosporin acylase and other enzymes. Crit. Rev. Biotechnol., 26: 95-120. DOI: $10.1080 / 07388550600718630$.

Srirangan, K., V. Orr, L. Akawi, A. Westbrook and M. Moo-Young et al., 2013. Biotechnological advances on penicillin $G$ acylase: Pharmaceutical implications, unique expression mechanism and production strategies. Biotechnol. Adv., 31: 13191332. DOI: 10.1016/j.biotechadv.2013.05.006

Suplatov, D., N. Panin, E. Kirilin, T. Shcherbakova and P. Kudryavtsev et al., 2014. Computational design of a ph stable enzyme: Understanding molecular mechanism of penicillin acylase's adaptation to alkaline conditions. PLoS ONE. DOI: 10.1371 /journal.pone.0100643

Suplatov, D.A., W. Besenmatter, V.K. Švedas and A. Svendsen, 2012. Bioinformatic analysis of alpha/beta-hydrolase fold enzymes reveals subfamily-specific positions responsible for discrimination of amidase and lipase activities. Protein Eng. Des. Sel., 25: 689-697.

DOI: $10.1093 /$ protein/gzs068

Szwajcer-Dey, E., J.R. Miller, S. Kovacevic and K. Mosbach, 1990. Characterization of a D-amino acid oxidase with high activity against cephalosporin $\mathrm{C}$ from the yeast trigonopsisvariabilis. Biochem. Int., 20: 1169-1178.

Voevodin, V., S.A. Zhumatiy, S.I. Sobolev, A. Antonov and S. Bryzgalov et al., 2012. Practice of "lomonosov" supercomputer. Open Syst. J., 7: 36-39.

Yamada, C., K. Kijima, S. Ishihara, C. Miwa and K. Wada et al., 2008. Improvement of the glutaryl-7aminocephalosporanic acid acylaseactivity of a bacterial gamma-glutamyltranspeptidase. Appl. Environ. Microbiol., 74: 3400-3409. DOI: 10.1128/AEM.02693-07

Zhang, W., Y. Liu, H. Zheng, S. Yang and W. Jiang. 2005. Improving the activity and stability of GL-7ACA acylase CA130 by site-directed mutagenesis. Appl. Environ. Microbiol., 71: 5290-5296. DOI: 10.1128/AEM.71.9.5290-5296.2005 